\title{
Sensitivity enhancement of chemically amplified resists and performance study using EUV interference lithography
}

\author{
Elizabeth Buitrago $^{1}$, Seiji Nagahara ${ }^{3}$, Oktay Yildirim ${ }^{2}$, Hisashi Nakagawa $^{6}$, Seiichi Tagawa ${ }^{4,5}$, \\ Marieke Meeuwissen $^{2}$, Tomoki Nagai ${ }^{6}$, Takehiko Naruoka ${ }^{6}$, \\ Coen Verspaget ${ }^{2}$, Rik Hoefnagels ${ }^{2}$, Gijsbert Rispens ${ }^{2}$, Gosuke Shiraishi ${ }^{7}$, Yuichi Terashita ${ }^{7}$, \\ Yukie Minekawa $^{7}$, Kosuke Yoshihara ${ }^{7}$, Akihiro Oshima ${ }^{4,5}$, Michaela Vockenhuber ${ }^{1}$ and Yasin \\ Ekinci $^{1}$ \\ ${ }^{1}$ Paul Scherrer Institute, CH-5232 Villigen PSI, Switzerland, \\ ${ }^{2}$ ASML Netherlands B.V., De Run 6501, 5504 DR Veldhoven, The Netherlands, \\ ${ }^{3}$ Tokyo Electron Ltd., Asaka Biz Tower, Tokyo 107-6325, Japan, \\ ${ }^{4}$ Graduate School of Engineering, Osaka University, Japan, \\ ${ }^{5}$ Institute of Scientific and Industrial Research, Osaka University, Ibaraki, Osaka 567-0047, Japan \\ ${ }^{6}$ JSR Corporation, Semiconductor Materials Laboratory, Fine Electronic Research Laboratories, 100 \\ Kawajiri-cho, Yokkaichi, Mie, 510-8552, Japan, \\ ${ }^{7}$ Tokyo Electron Kyushu Ltd., 1-1, Fukuhara, Koshi-shi, Kumamoto 861-1116, Japan \\ SPIE Advanced Lithography 2016, Extreme Ultraviolet (EUV) Lithography VI \\ Keywords: EUVL, IL, CAR, photoresist, sensitivity enhancement, $7 \mathrm{~nm}$ node, $5 \mathrm{~nm}$ node
}

\begin{abstract}
Extreme ultraviolet lithography (EUVL, $\lambda=13.5 \mathrm{~nm}$ ) is the most promising candidate to manufacture electronic devices for future technology nodes in the semiconductor industry. Nonetheless, EUVL still faces many technological challenges as it moves toward high-volume manufacturing (HVM). A key bottleneck from the tool design and performance point of view has been the development of an efficient, high power EUV light source for high throughput production. Consequently, there has been extensive research on different methodologies to enhance EUV resist sensitivity. Resist performance is measured in terms of its ultimate printing resolution, line width roughness (LWR), sensitivity (S or best energy BE) and exposure latitude (EL). However, there are well-known fundamental trade-off relationships (LRS trade-off) among these parameters for chemically amplified resists (CARs). Here we present early proof-of-principle results for a multi-exposure lithography process that has the potential for high sensitivity enhancement without compromising other important performance characteristics by the use of a Photosensitized Chemically Amplified Resist $^{\mathrm{TM}}\left(\mathrm{PSCAR}_{\mathrm{TM}}\right)$. With this method, we seek to increase the sensitivity by combining a first EUV pattern exposure with a second UV flood exposure $(\lambda=365 \mathrm{~nm})$ and the use of a PSCAR. In addition, we have evaluated over 50 different state-of-the-art EUV CARs. Among these, we have identified several promising candidates that simultaneously meet sensitivity, LWR and EL high performance requirements with the aim of resolving line space (L/S) features for the 7 and $5 \mathrm{~nm}$ logic node (16 nm and $13 \mathrm{~nm}$ half-pitch HP, respectively) for HVM. Several CARs were additionally found to be well resolved down to $12 \mathrm{~nm}$ and $11 \mathrm{~nm}$ HP with minimal pattern collapse and bridging, a remarkable feat for CARs. Finally, the performance of two negative tone state-of-the-art alternative resist platforms previously investigated was compared to the CAR performance at and below $16 \mathrm{~nm}$ HP resolution, demonstrating the need for alternative resist solutions at $13 \mathrm{~nm}$ resolution and below. EUV interference lithography (IL) has provided and continues to provide a simple yet powerful platform for academic and industrial research enabling the characterization and development of new resist materials before commercial EUV exposure tools become available. Our experiments have been performed at the EUV-IL set-up in the Swiss Light Source (SLS) synchrotron facility located at the Paul Scherrer Institute (PSI).
\end{abstract}

Extreme Ultraviolet (EUV) Lithography VII, edited by Eric M. Panning, Kenneth A. Goldberg. Proc. of SPIE Vol. 9776, 97760Z · C 2016 SPIE · CCC code: 0277-786X/16/\$18 · doi: 10.1117/12.2220026 


\section{INTRODUCTION}

Although there still no clear or set technology path, EUVL is predicted to be introduced in HVM at the $7 \mathrm{~nm}$ node according to ASML's (September 2015) NXE extension roadmap [1]. With the successful demonstration of the first $7 \mathrm{~nm}$ node test chips by the use of silicon germanium transistor technology in the second half of 2015, IBM Research has furthermore demonstrated the feasibility of introducing EUVL in HVM at multiple lithography steps [2]. EUVL is still therefore the most promising lithography candidate to manufacture electronic devices in the now near future and the availability of high performance EUVL materials to resolve features down to $16 \mathrm{~nm}$ and $13 \mathrm{~nm}$ HP is ever more important.

ASML has reported remarkable dose control EUV light source power up to $183 \mathrm{~W}$ in 2015 [1]. Nonetheless, it is clear that EUV source power remains a bottleneck for high throughput manufacturing. For that reason, here we have focused on finding the best CAR candidates from vendors from around the world with particularly high sensitivities $\left(\mathrm{BE}<30 \mathrm{~mJ} / \mathrm{cm}^{2}\right.$ at $16 \mathrm{~nm} \mathrm{HP}$ and $<50 \mathrm{~mJ} / \mathrm{cm}^{2}$ at $13 \mathrm{~nm} \mathrm{HP}$ ) for the $7 \mathrm{~nm}$ and $5 \mathrm{~nm}$ logic node while still maintaining other high performance characteristics (LWR and EL). Also, the performance of two state-of-the-art alternative resist platforms (Sn-based metal organic resist from Inpria Corp. and a negative tone CAR molecular resist from Irresistible Materials) previously investigated [3-4] was compared to the CAR performance at and below $16 \mathrm{~nm}$ HP resolution for HVM demonstrating the need for alternative resist solutions at $13 \mathrm{~nm}$ resolution and below.

In this report we also present proof-of-principle results for a multi-exposure lithography process that has the potential for high sensitivity enhancement without compromising other important performance characteristics such as LWR and EL as will be shown later in this report. With this novel method, we aim at a resist sensitivity enhancement by the combination of a first EUV pattern exposure, a second UV flood exposure and the use of a photosensitized chemically amplified resist (PSCAR ${ }_{\mathrm{TM}}$ ) [5-6]. The PSCAR concept was introduced by Tagawa et al., [5-6] from Osaka University in Japan by the use of a first pattern exposure by electron beam lithography (EBL). By the combination of a first EBL pattern exposure and a second UV flood exposure the authors found a sensitivity increase of a factor of 10 without any loss of resolution [5-6] down to $75 \mathrm{~nm}$ for L/S patterns demonstrating high potential for resist sensitivity improvement for high resolution patterning as will be explored in the following sections.

The performance of the different photoresists was evaluated using EUV interference lithography (EUV-IL) at the Swiss Light synchrotron facility located at the Paul Scherrer Institute [7]. With EUV-IL, a mask with transmission-diffraction gratings is illuminated by a spatially coherent beam of EUV light $(\lambda=13.5 \mathrm{~nm})$ [8-9]. Periodic images can then be produced by the interference of two or more diffracted beams. First-order diffracted beams overlap at a distance from the mask where the aerial image is created. The produced image has a period $P$ that is given as (Eq. 1):

$$
P=\frac{\lambda}{2 \sin \theta_{m}}=\frac{P_{g}}{2 m}
$$

where $\theta_{m}$ denotes the diffraction angles and $m$ the diffraction order, and $P_{g}$ is mask grating period, that is half of the mask grating period $P_{g}$ when a pair of first-order $(m=1)$ diffraction interference beams are used. With this technique it is therefore possible to create high-resolution periodic images for the fast characterization of EUV photoresists [7]. In fact, using the EUV-IL tool at PSI it has been possible to resolve patterns down to $7 \mathrm{~nm}$ in resolution, the record for photonbased lithography [7, 10-12]. EUV-IL therefore provides a simple and powerful platform for academic and industrial research while the availability of high resolution commercial EUV exposure tools still limited. The aerial image created by interference lithography is a sinusoidal image which is similar to that of a scanner with dipolar illumination at its NA limited HP.

\section{METHODS}

The transmission-diffraction masks used here consist of HSQ (hydrogen silsesquioxane) gratings fabricated on 100-nmthick $\mathrm{Si}_{3} \mathrm{~N}_{4}$ membranes and a gold photon-stop covering all areas around the gratings to absorb the zeroth-order beam. The fabrication process is explained in more detail elsewhere [9]. Each mask includes three pairs of gratings for every pitch. Six different pitches are included in every mask. For this study a single mask (M1) that includes pairs of gratings 
to pattern with $11 \mathrm{~nm}, 12 \mathrm{~nm}, 13 \mathrm{~nm}, 14 \mathrm{~nm}, 16 \mathrm{~nm}$ and $18 \mathrm{~nm}$ HP resolution on wafer was used unless otherwise specified.

Dose-on-mask $\left(\mathrm{mJ} / \mathrm{cm}^{2}\right)$ is determined from the measured flux $\left(\mathrm{mW} / \mathrm{cm}^{2}\right.$, measured before and after the exposure) and exposure time (s). The corrected dose-on-wafer values are calculated based on predetermined tool-factors (ratio between the dose-on-mask and dose-on-wafer) proportional to the mask efficiency [13]. The mask efficiency defined as (Eq. 2):

$$
\eta=D_{O F} / D_{50 \%}
$$

is dependent on the diffraction efficiency of the gratings and the nitride membrane transparency (transmission efficiency) [8]. $D_{O F}$ is the $50 \%$ clearing dose through an open frame and $D_{50 \%}$ is the $50 \%$ clearing dose per grating pair on mask from the first-order non-interfering diffraction patterns. The tool-factors are pitch and grating dependent.

To determine the mask efficiency per grating pair needed to determine the mask tool factors, an exposure through an open frame $\left(1.5 \times 1.5 \mathrm{~mm}^{2}\right)$ is first performed at different doses using a reference resist (UL1R12) to determine the $50 \%$ clearing dose $D_{O F}$. After development, the thickness of the resist is measured as a function of dose. A contrast curve is then plotted as the normalized remaining film thickness after development $v s$. log exposure dose. The normalized thickness is calculated as the film thickness that remains after development divided by the initial film thickness before development. The $50 \%$ clearing dose through the open frame $\left(D_{O F}=\log D_{0}\right)$ can then be determined from the contrast curve when fitted by a dose-response function (Eq. 3):

$$
t(D)=t_{0}\left[1+10^{\left(\log D_{0}-D\right) \times \gamma}\right]^{-1}
$$

Where $D$ is the exposure dose-on-mask, $t_{0}$ is the maximum thickness difference of unexposed or overexposed resist, $\log D_{0}$ is the dose for $50 \%$ clearance and $\gamma$ is the slope (contrast) [7-8].

Another dose scan with the same reference resist (UL1R12) is then performed using the actual mask for which the toolfactors need to be determined in order to calculate the mask efficiency $\eta$ per grating pair. The resist thickness after development resulting from the first-order non-interfering diffraction is recorded as a function of dose-on-mask. The $50 \%$ clearance dose $\left(D_{50 \%}\right)$ is found from the normalized thickness as function of dose-on-mask data when fitted by a dose-response function as described previously.

The Z-factor is a global performance figure of merit that generally accounts for the RLS trade-off relationship and should be used with caution. Nonetheless, it enables a straightforward and fast comparison of different resists and resists platforms. Here we use the simplified approach from Wallow et al., [14] in which the so called $Z$-factor is defined as (Eq. 4):

$$
Z=\left(\text { Sensitivity) } \times(L E R)^{2} \times(H P)^{3}\right.
$$

The best energy (BE) represents the sensitivity.

The exposure latitude is calculated from the average critical dimension $(C D)$ vs. exposure dose plots. A linear curve is fitted through the points within the $C D_{\max }$ and $C D_{\min }$ values (or critical dimension $+10 \%$ or $-10 \%$ respectively). $E n C D_{\max }$ and $E n C D_{\min }$ (exposure dose at $C D+10 \%$ and $C D-10 \%$ respectively) are extracted from the linear fit. The maximum exposure latitude $E L_{\max }$ is defined as (Eq. 5):

$$
E L_{\max }=\left|\left(E n C D_{\min }-E n C D_{\max }\right) / B E \times 100\right|
$$

The actual exposure latitude $E L_{a c t}$ that is reported here is furthermore determined by instead using the minimum and maximum exposure dose values ( $E n C D_{\max , a c t}$ and $E n C D_{\min , a c t}$ respectively) within $E n C D_{\max }$ and $E n C D_{\min }$ for which 
no pinching, necking or pattern collapse, etc., is observed. The actual, line quality driven exposure latitude $E L_{a c t}$ may therefore be lower than the $E L_{\max }$.

SEM images of the resist patterns were obtained using a SEM Carl Zeiss SUPRA 55VP. The in-lens detector with a scanning speed of 10 seconds was used. The acceleration voltage and aperture size was set to $1 \mathrm{kV}$ and $7.5 \mu$ respectively to minimize electron beam damage and get surface information. The magnification and working distance are both kept constant at 400000 and $\sim 3 \mathrm{~mm}$, respectively. The CD and LER values are obtained from the analysis performed with a commercially available modeling software (SuMMIT $\left.{ }^{\circledR}\right)$.

In this study, 97 EUV-IL exposures were performed over a period of one year (2015) to test 52 different state-of-the-art positive tone CARs from different vendors all around the world. The naming convention used here is consistent (i.e., $\mathrm{UL} 1=$ underlayer $1, \mathrm{R} 1=$ resist 1$)$. Different underlayer formulations from different vendors may also have been used. The type of resist or underlayer and process conditions (resist and underlayer thickness, post-exposure bake (PEB), postapply bake (PAB) temperature and process time) were either determined by the different resist suppliers, or their effect on resist performance was as well subject to investigation. Resist development was performed in a semiautomatic SUSS Microtech tool that includes a 30 second 2.38\% TMAH dispense step. The type of development used here was the same unless otherwise noted. Reference exposures using our reference resist and underlayer material R1UL1 were regularly performed (14 repetitions).

\section{LONG TERM PERFORMANCE OF EUV-IL TOOL AT PSI}

The long term performance and stability of the EUV-IL tool at PSI is continuously improved and monitored by performing an exposure with a baseline resist process UL1R12. The same mask, beam conditions and resist processing parameters are used for all exposures. Fig. 1 shows the BE, average LWR and average EL values obtained from exposures performed within an 11 month period from February to December 2015. The average and standard deviation of the BE, LWR and EL for $16 \mathrm{~nm}$ and $22 \mathrm{~nm}$ are also shown in the corresponding graphs. The standard deviation of the best energy at $22 \mathrm{~nm} \mathrm{HP}$ is below 7\% which is an improvement from the value that was reported in $2013 \sim 10 \%$ [7]. For the last six months of the year the BE variation is furthermore improved with a standard deviation $<3 \%$ at $22 \mathrm{~nm} \mathrm{HP}$ which is $<1.5 \%$ of the mean. This corresponds to changes that were made to our mirror cooling system, which now operates at lower temperatures since the summer of 2015 and thereby improved the optical stability.

It is clear from the graphs that there is a certain degree of variation from exposure to exposure which may arise from the actual EUV-IL tool, the SEM conditions, or even resist aging. The SEM image analysis is the main source of variation to the LWR values reported. The SEM inspection is manually performed using a multipurpose, multi-user SEM in a research environment. Though the same SEM settings are used and the analyis is always performed the same way, the SEM is only serviced due to failure and human error is unavoidable as the SEM picture acquisition and analysis is executed manually. We also note that the LWR values reported in this work are consistently higher by $\sim 30 \%$ than those reported elsewhere for the same resists and processing conditions. Therefore one should take the LWR values not as universal values, but as an in-house number. Nevertheless, the parameters are kept constant for all our analysis so that it enables a true comparison of the different resists. 

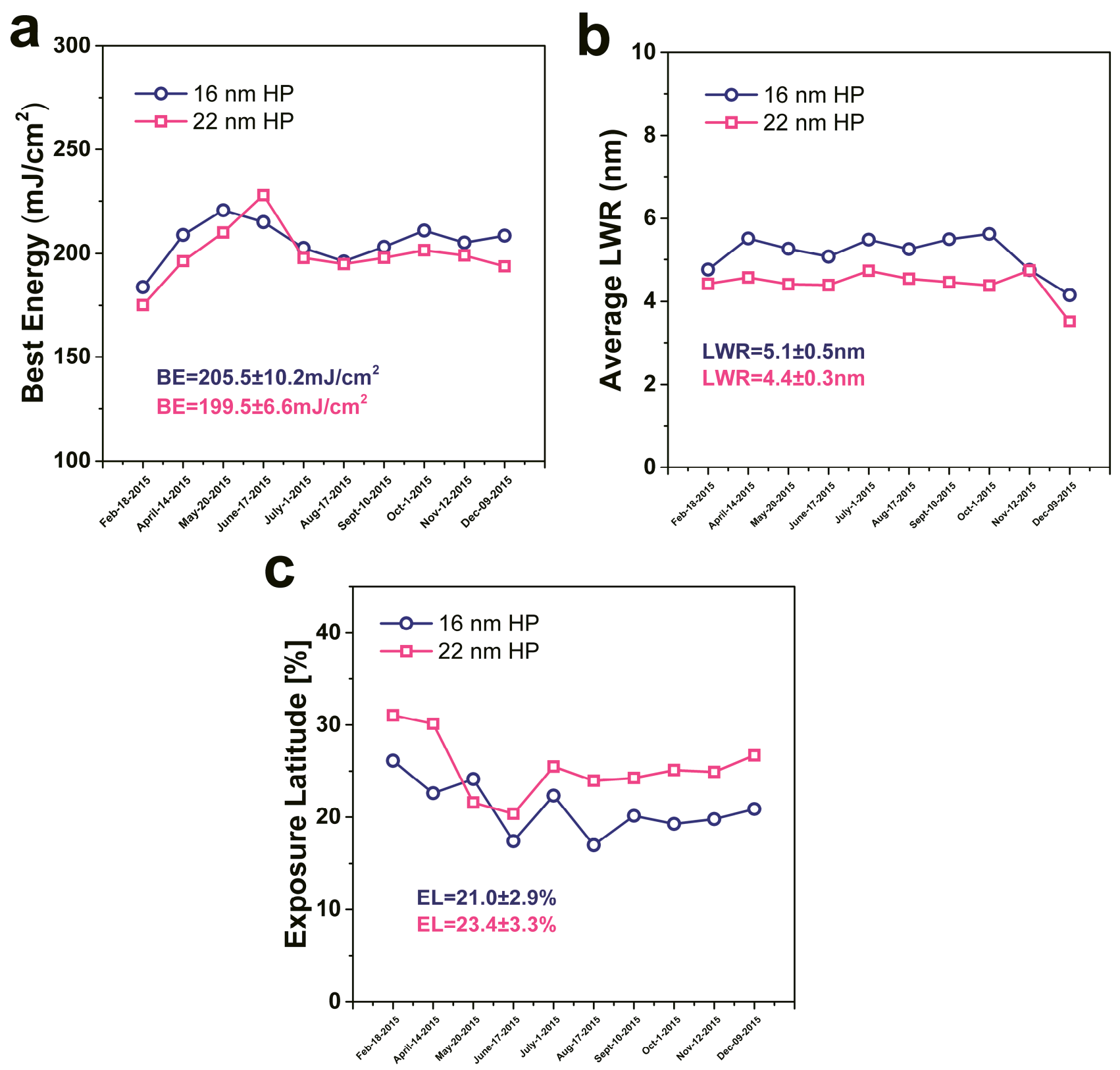

Figure 1. (a) BE, (b) Average LWR and (c) EL over time for an 11 month period (February to December) in 2015 for $16 \mathrm{~nm}$ and $22 \mathrm{~nm}$ HP using a reference resist process UL1R12.

\section{CAR EVALUATION AND COMPARISON AT 16 AND 13 NM RESOLUTION AND BEYOND}

Within this project, over 50 different state-of-the-art positive tone CARs have been evaluated by EUV-IL with the aim to resolve (L/S) patterns down to $16 \mathrm{~nm}$ and $13 \mathrm{~nm}$ HP for HVM. The graphs presented in Fig. 2 show LER vs. BE (dose-to-size values for 1:1 L/S patterns) values determined at (a) $16 \mathrm{~nm}$ and (b) $13 \mathrm{~nm}$ HP. For comparison purposes, the LER vs. BE values obtained from two high performing negative tone state-of-the-art alternative resist platforms (Sn-based metal organic resist from Inpria Corp. at $25 \mathrm{~nm}$ and $32 \mathrm{~nm}$ thickness and two xMT molecular resists from Irresistible Materials: xMT-0801 and xMT-0614) previously investigated [3-4] were plotted in the same graphs. The solid curves cross the dots with minimum $Z$-factors obtained at the corresponding HP. 

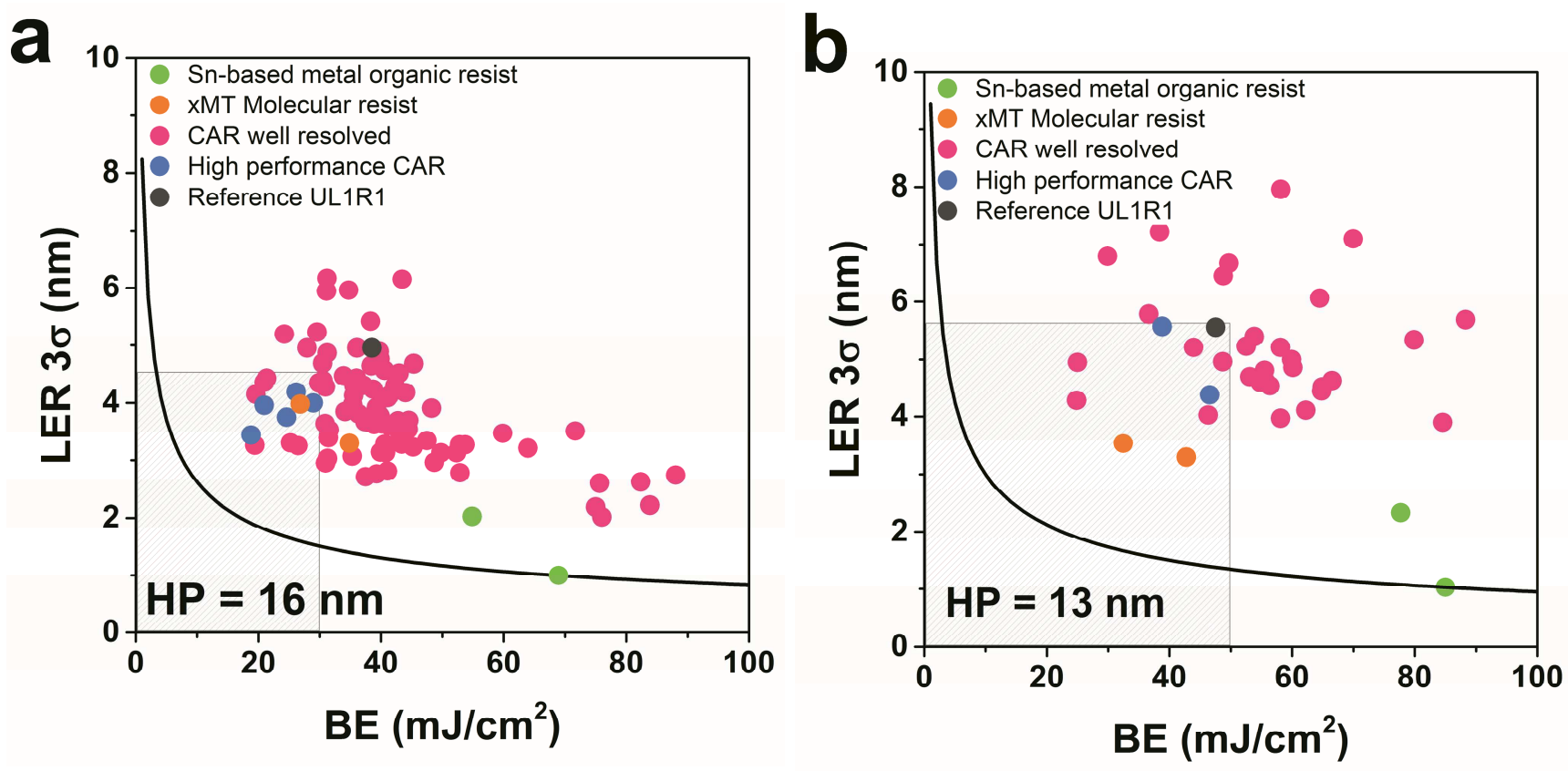

Figure 2. LER vs. BE values obtained from over 95 exposures using 52 different chemically amplified resists at (a) $16 \mathrm{~nm}$ and (b) $13 \mathrm{~nm}$ HP. Every dot represents exposures for which the resist was well resolved down to the appropriate pitch. The solid curves in the same graphs represent the constant $Z$-factors crossing the dots with minimum $Z$-factors at the corresponding HPs. Best performing CARs with BE $<30 \mathrm{~mJ} / \mathrm{cm}^{2}$, LWR $<6.5 \mathrm{~nm}$ (LER $<4.6 \mathrm{~nm}$ ) and EL $>15 \%$ are represented by purple dots (these are arbitrary threshold values, not industry targets). The LER vs. BE values obtained from a Sn-based metal organic resist from Inpria Corp. at two different thicknesses (25 and $32 \mathrm{~nm}$ ) and two molecular resists xMT from Irresistible Materials were also plotted in the same graphs for comparison purposes.
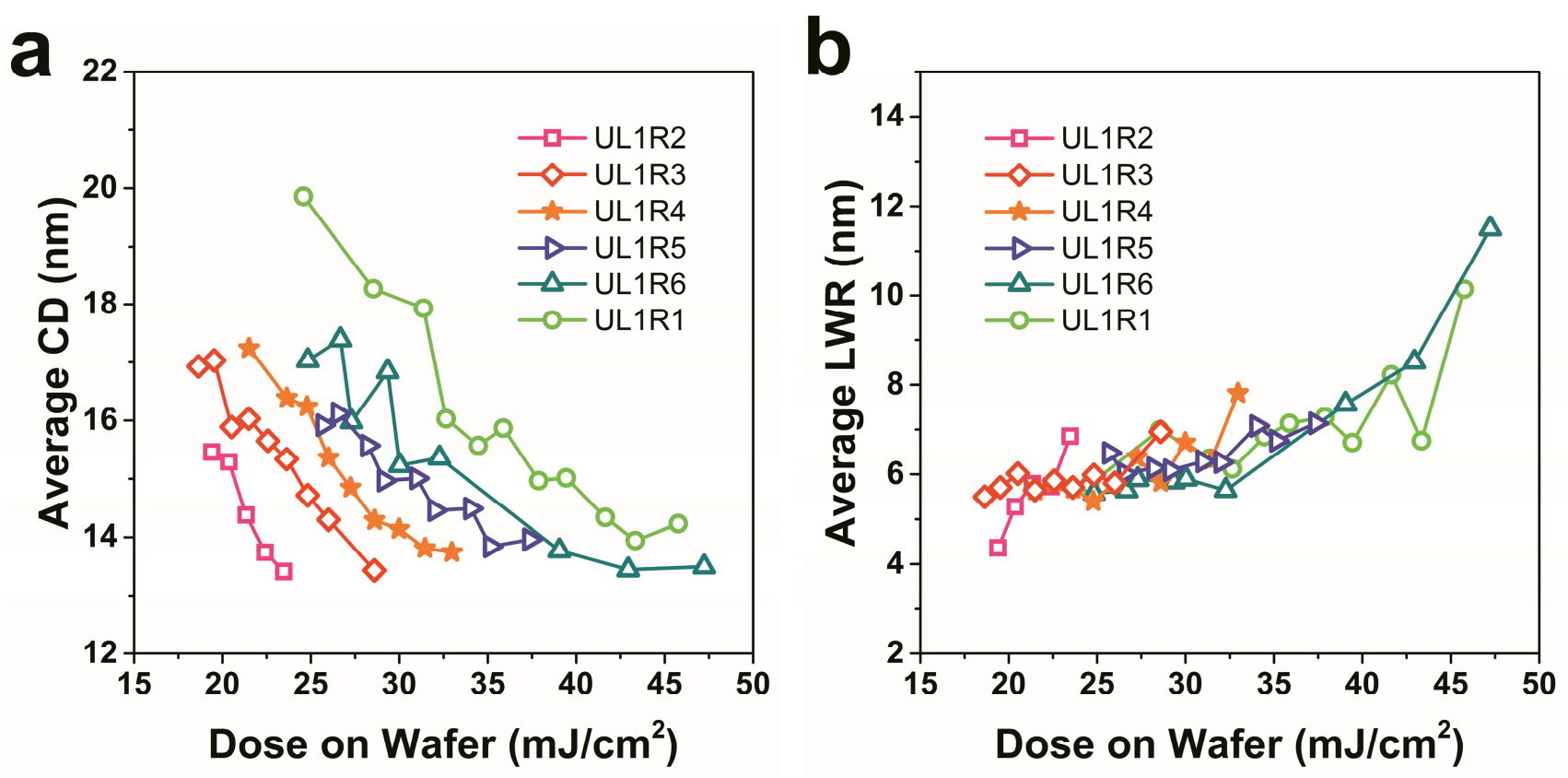

Figure 3. Average CD vs. dose (a) and average LWR vs. dose (b) graphs for the highest performing resists (R2-R6) tested (BE values $<30 \mathrm{~mJ} / \mathrm{cm}^{2}, \mathrm{LWR}<6.5 \mathrm{~nm}$ and EL $>15 \%$, these are arbitrary threshold values not industry targets) at $16 \mathrm{~nm}$ HP resolution. UL1R1 is the reference resist. 
Table 1. BE, EL, LER and Z-factors for the best performing CARs (R1-R5) at $\mathrm{HP}=16 \mathrm{~nm}$. The same UL material was used. UL1R1 is the reference resist process.

\begin{tabular}{|l|c|c|c|c|}
\hline Name & $\begin{array}{c}\text { BE } \\
(\mathrm{mJ} / \mathrm{cm} 2)\end{array}$ & EL (\%) & $\begin{array}{c}\text { LWR } \\
(\mathbf{n m})\end{array}$ & z-factor \\
\hline UL1R2 & 18.8 & 16.0 & 5.6 & $9.1 \mathrm{E}-09$ \\
\hline UL1R3 & 20.9 & 25.9 & 5.9 & $1.3 \mathrm{E}-08$ \\
\hline UL1R4 & 24.6 & 19.5 & 5.5 & $1.4 \mathrm{E}-08$ \\
\hline UL1R5 & 26.2 & 24.1 & 6.4 & $1.9 \mathrm{E}-08$ \\
\hline UL1R6 & 29.0 & 17.3 & 5.9 & $1.9 \mathrm{E}-08$ \\
\hline UL1R1 (avg)-ref & $38.5 \pm 6.3$ & $22.6 \pm 5.2$ & $6.5 \pm 1.3$ & $2.9 \mathrm{E}-08$ \\
\hline
\end{tabular}

Fig. 2a shows LER vs. BE (dose-to-size values for 1:1 L/S patterns) values determined from well resolved patterns at $16 \mathrm{~nm}$ HP. Each dot represents an exposure for which a resist was well resolved down $16 \mathrm{~nm}$. In fact, 47 different CARs are well resolved down to $16 \mathrm{~nm}$ HP. Nonetheless, resolution alone is not enough for HVM and the best performing resists need to perform well in terms of BE, LWR and EL simultaneously. Here, 5 different CARS are highly performing with $\mathrm{BE}<30 \mathrm{~mJ} / \mathrm{cm}^{2}, \mathrm{LWR}<6.5 \mathrm{~nm}(\mathrm{LER}<4.6 \mathrm{~nm})$ and EL $>15 \%$. The LWR, BE and EL threshold values used here to pick the best resist candidates are arbitrary values not set by industry. They are represented by purple dots in Fig $2 \mathrm{a}$. From Table 1 and Fig. 3a and 3b it is clear that UL1R2, UL1R3 and UL1R4 are in particular interesting for HVM having the highest sensitivities (BE values $<25 \mathrm{~mJ} / \mathrm{cm}^{2}$ ) and the lowest LWR values $<6 \mathrm{~nm}$. From these resists, UL1R3, UL1R4 and UL1R5 furthermore have small EL $>9 \%$ at $14 \mathrm{~nm}$ HP with small BE $(22.6-28.2 \mathrm{~mJ} / \mathrm{cm} 2)$ and small LWR values $(6.5-7.1 \mathrm{~nm})$ making these materials also highly promising down to $14 \mathrm{~nm}$ resolution. UL1R3 does not only have the lowest $Z$-factor among these high performing resists due to its low BE and LWR values but it also has an extremely high exposure latitude of over $25 \%$. We have therefore identified highly competitive CAR candidates necessary for the $7 \mathrm{~nm}$ node and the successful introduction of EUVL into HVM. Fig. 4 shows line/space SEM images at different doses around the BE for some of the best performing CARs and reference UL1R1 at $16 \mathrm{~nm} \mathrm{HP}$ resolution.

From the alternative resist platforms considered here at $16 \mathrm{~nm}$ HP, molecular resist xMT-0801 was also found to be high performing with a BE $<30 \mathrm{~mJ} / \mathrm{cm}^{2}$, LWR $<6.5 \mathrm{~nm}(\mathrm{LER}<4.6 \mathrm{~nm})$ and EL $>15 \%$. Furthermore, the Sn-based metal organic resist from Inpria Corp. tested at two different thicknesses (25 and $32 \mathrm{~nm})$ has the smallest $Z$-values as can be seen in Fig. 2a even when exhibiting high BE values due to their extraordinarily low LWRs $<3.1 \mathrm{~nm}$. The Sn-based metal organic resist also shows extraordinarily high exposure latitude values of over $30 \%$ at $16 \mathrm{~nm}$ resolution with either thickness tested. As previously mentioned, Fig. $2 \mathrm{~b}$ also shows LER $v s$. BE values determined from well resolved patterns at $13 \mathrm{~nm}$ HP. 17 different CARs are well resolved down to $13 \mathrm{~nm}$ HP. The high performance arbitrary threshold values (not set by industry) requirements that need to be met simultaneously at such high resolution have been slightly relaxed and three high performing candidates have been determined. The best CAR candidates at $13 \mathrm{~nm}$ resolution simultaneously show $\mathrm{BE}<50 \mathrm{~mJ} / \mathrm{cm}^{2}, \mathrm{LWR}<8 \mathrm{~nm}(\mathrm{LER}<5.6 \mathrm{~nm})$ and $\mathrm{EL}>3 \%$ as shown in Table 2.

Table 2. BE, EL, LWR and $Z$-factors for the best performing CAR resists at $\mathrm{HP}=13 \mathrm{~nm}$.

\begin{tabular}{|l|c|c|c|c|}
\hline Name & $\begin{array}{c}\text { BE } \\
(\mathbf{m J} / \mathrm{cm} 2)\end{array}$ & EL (\%) & $\begin{array}{c}\text { LWR } \\
(\mathbf{n m})\end{array}$ & z-factor \\
\hline UL1R7 & 38.8 & 8.8 & 7.9 & $2.6 \mathrm{E}-08$ \\
\hline UL1R8 & 46.6 & 4.9 & 6.6 & $2.0 \mathrm{E}-08$ \\
\hline UL1R1 -ref & 47.5 & 3.2 & 7.9 & $3.2 \mathrm{E}-08$ \\
\hline
\end{tabular}

In Fig. 5 we can see the (L/S) SEM pictures at different doses around the BE for the best performing CARs at $13 \mathrm{~nm}$. Pattern collapse, bridging and pinching, in particular limits the EL at $13 \mathrm{~nm}$ resolution and below. Though the performance of CARs at $13 \mathrm{~nm}$ HP and below still needs to be improved, it is worth noting that to our knowledge, it is the first time that any exposure latitude has been observed at such high resolutions for a CAR. It is nonetheless clear from these results that there is an impending need for alternative resist solutions at $13 \mathrm{~nm}$ resolution and below. Again, even with high BE values, the Sn-based metal organic resists (25 and $32 \mathrm{~nm}$ thickness) shows the smallest $Z$-values and the highest EL $(>10 \%)$ and lowest LWR values $(<3.5 \mathrm{~nm})$ at this HP as shown in Fig. $2 \mathrm{~b}$. 


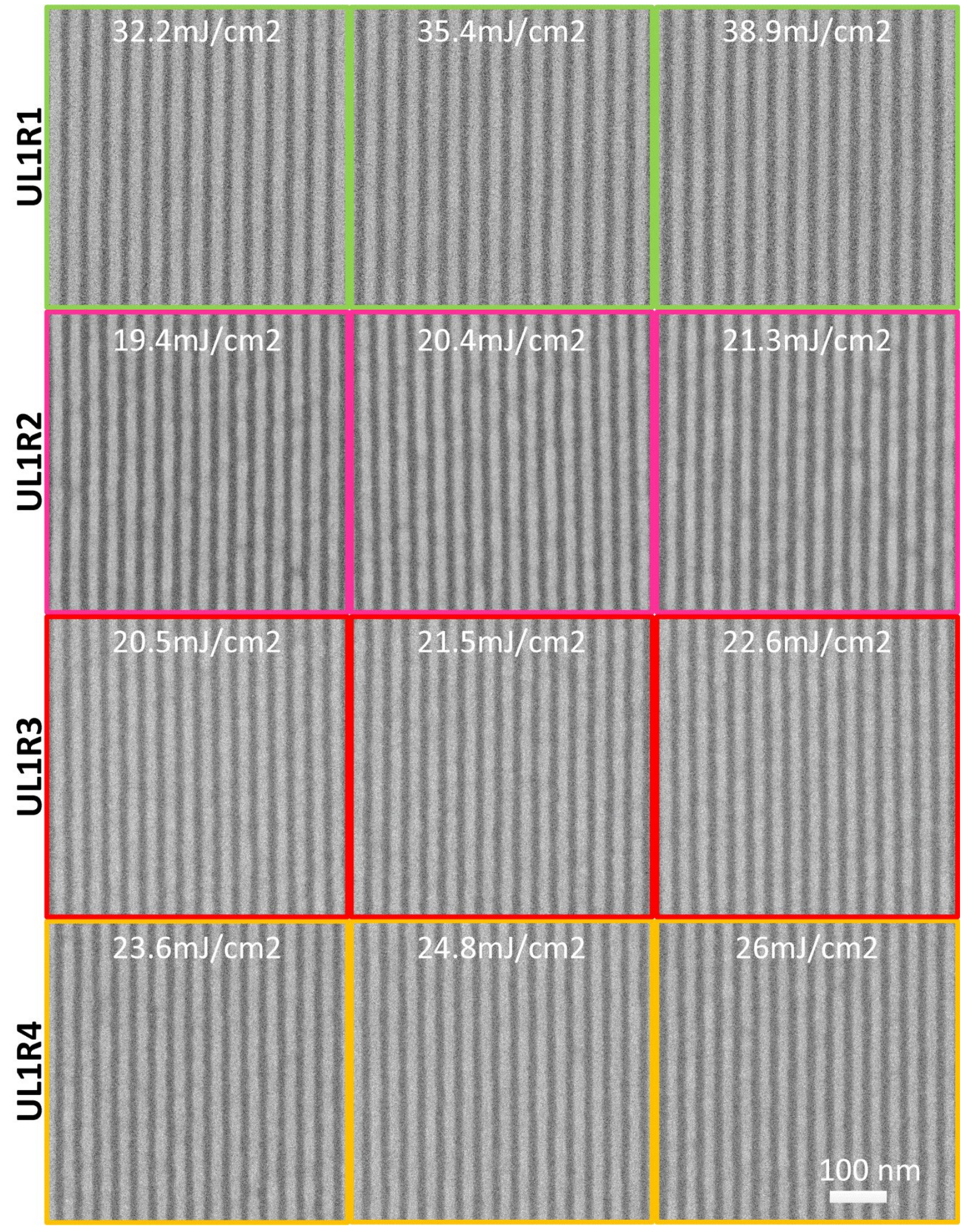

Figure 4. SEM (L/S) images showing some of the highest performing resists (R2-R4) tested (BE values $<30 \mathrm{~mJ} / \mathrm{cm}^{2}$, LWR $<6.5 \mathrm{~nm}$ and $\mathrm{EL}>15 \%$ ) at $16 \mathrm{~nm}$ HP resolution. UL1R1 is the reference resist. 

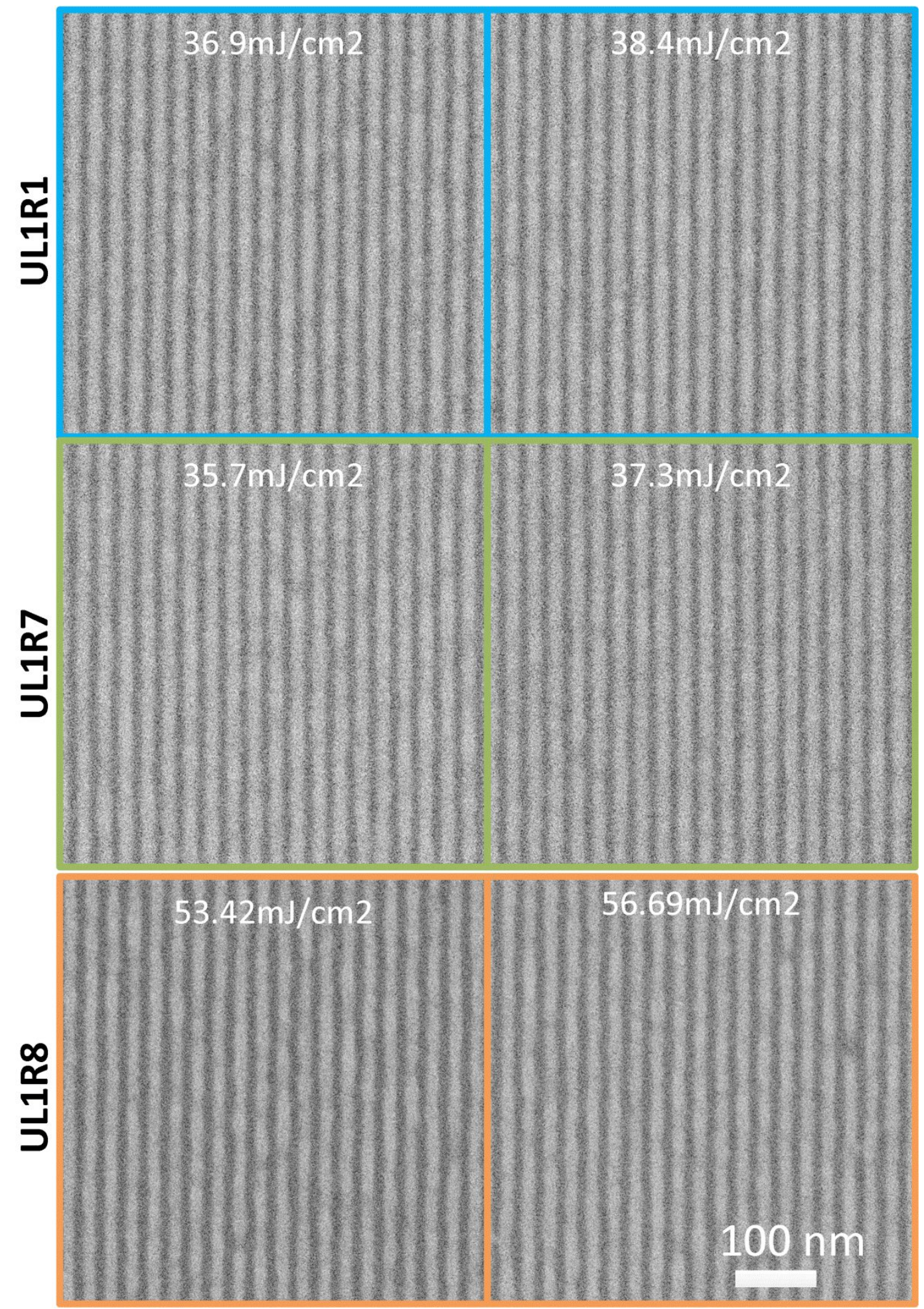

Figure 5. SEM (L/S) images showing some of the highest performing resists (R1, R7 and R8) tested (BE values $<50 \mathrm{~mJ} / \mathrm{cm}^{2}, \mathrm{LWR}<8 \mathrm{~nm}$ and $\mathrm{EL}>3 \%$ ) at $13 \mathrm{~nm} \mathrm{HP}$ resolution. 
We have furthermore found three different CAR resists that are well resolved down to $12 \mathrm{~nm} \mathrm{HP}$ and can be resolved down to $11 \mathrm{~nm}$ HP with slight pattern collapse and bridging, as can be seen in Fig. 5. Considering that only last year only one CAR UL1R1 was found to be well resolved down to $12 \mathrm{~nm}$ this is a remarkable feat for CARs [9].
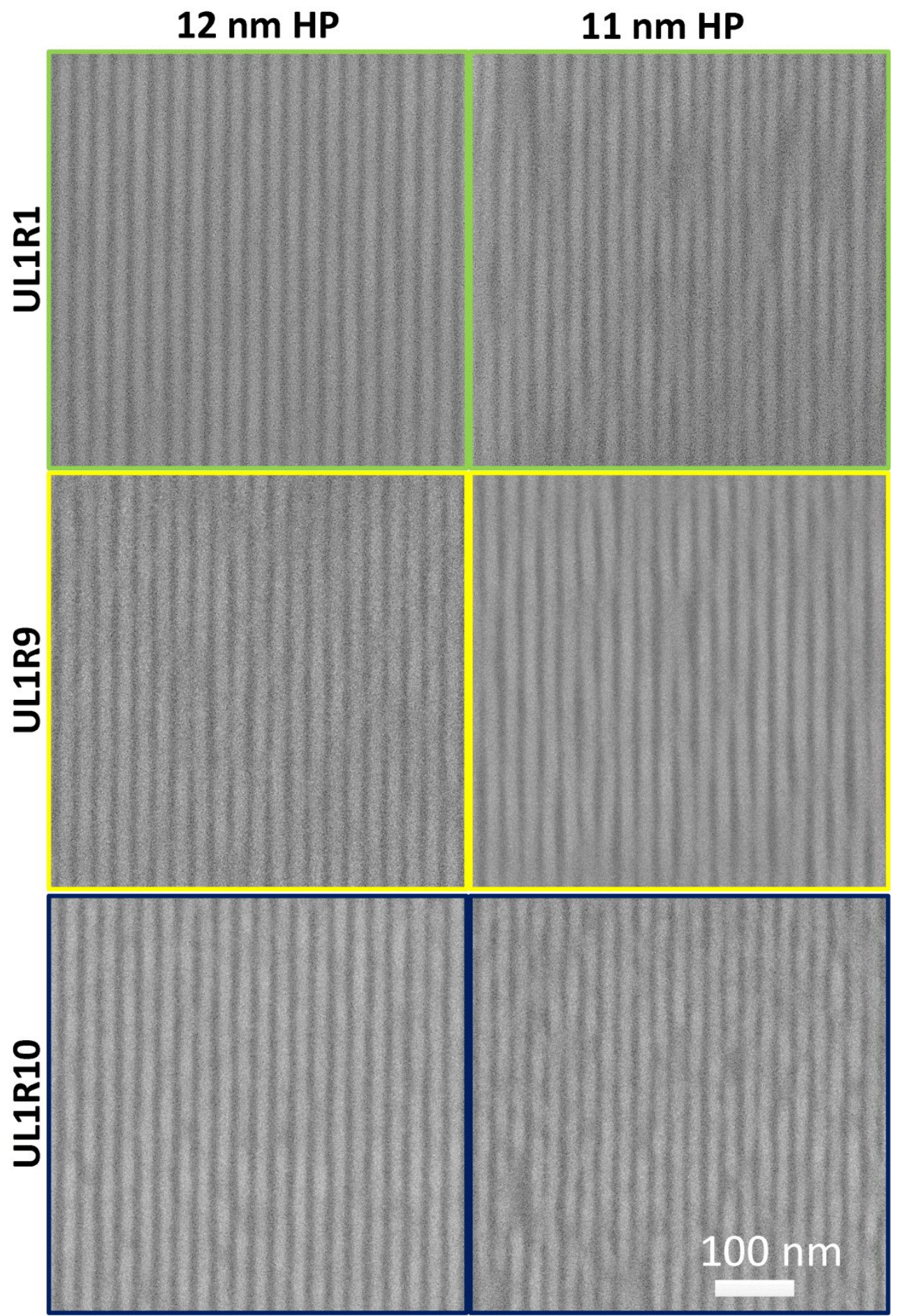

Figure 6. SEM (L/S) images showing some the highest performing resists at 12 and $11 \mathrm{~nm} \mathrm{HP}$ resolution. Minimal pattern collapse and bridging are observed. 


\section{PSCAR $_{T M}$ PROCESS}

Ito H. et al., [15] introduced the chemical amplification concept (by means of an acid catalyst) for the first time in the early 1980's. Since its inception, chemical amplification has been the base upon which state-of-the-art photoresist technology has been continuously designed and developed from. Nonetheless, there are well known fundamental tradeoff relationships (LRS trade-off) among the LWR, resolution and sensitivity that limit the ultimate performance of CARs $[14,16]$. Therefore, extensive research continues on different methodologies to enhance EUV resist sensitivity.

In the conventional chemical amplification process, the amount of photogenerated acid produced increases with exposure dose. Acid contrast (resist contrast) is also enhanced by increasing the exposure dose and quencher (Q) and acid amplification happens during the post exposure bake (PEB). Tagawa et al., [5-6] proposed a new sensitization enhancement mechanism in which acid amplification also happens by non-thermal diffusion processes at room temperature. The PSCAR $\mathrm{TM}_{\mathrm{TM}}$ is a multi-exposure (Figure 1) lithography technique in which a low-power pattern exposure (i.e., EUV) is followed by a high-power UV flood exposure $(\lambda=365 \mathrm{~nm})$ while using a PSCAR. In this PSCAR process, a photosensitizer and an acid are produced during the first low-power pattern exposure. Then, a large amount of acid is produced through the photo-excitation of the photosensitizer during the second high-power UV-flood exposure in the initially exposed area only. In comparison to the conventional CAR process where acid generation happens during the EUV exposure (or ELB) alone, in the PSCAR process, a second acid generation process takes place during the second UV-flood exposure [5-6]. The goal is to increase the initial acid yield produced during the first low-power EUV pattern exposure while maintaining the same acid distribution during the second UV flood exposure to overcome RLS trade-off of conventional CARs. The PSCAR should have no absorption in the wavelength region of the flood exposure so that the acid profile generated by second UV-flood exposure is identical to profile of the PS generated by first pattern exposure. Acid production during the second UV-flood exposure then should only occur by the excitation of the photosensitizer and non-thermal diffusion.

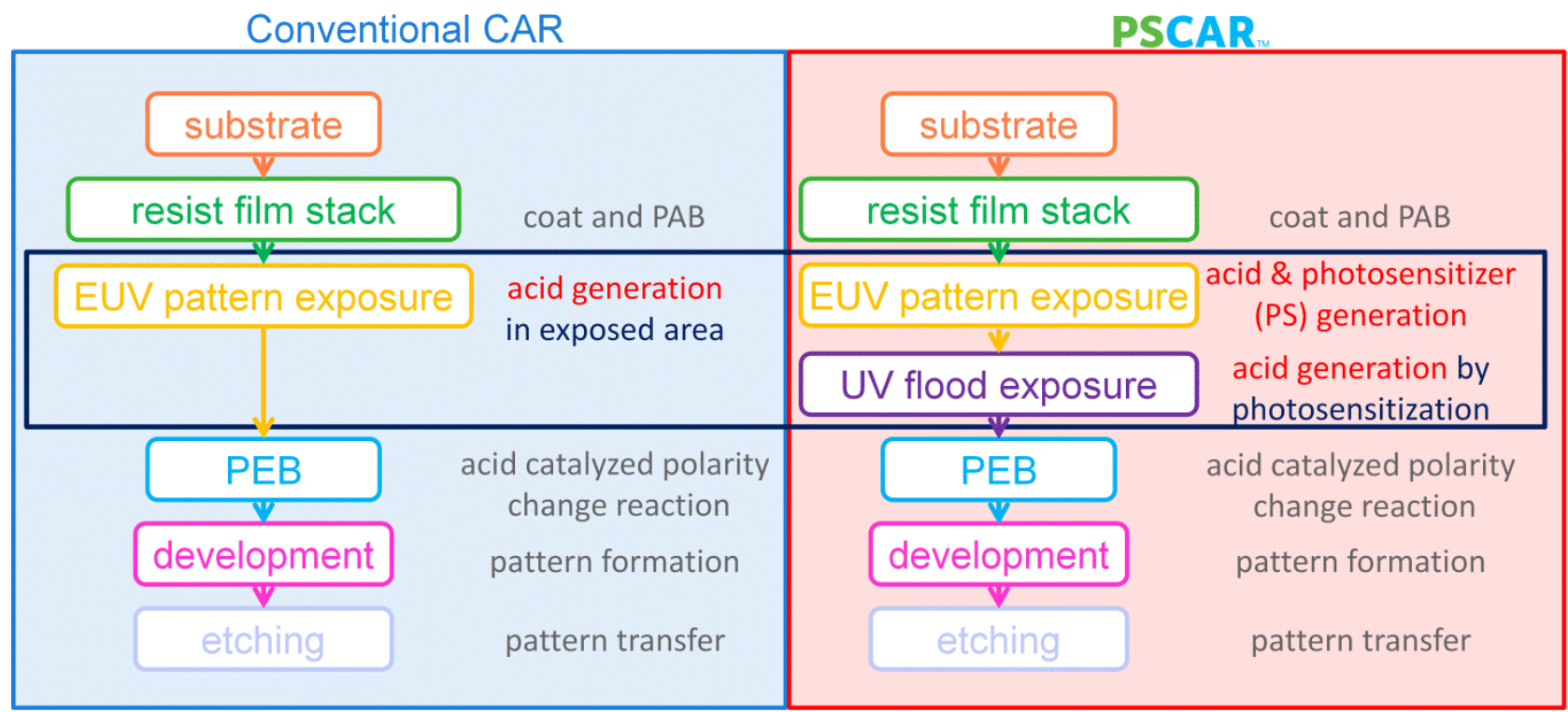

Figure 7. Comparison of PSCAR concept and conventional CAR processes.

In this work we have obtained early proof-of-principle results by performing a first EUV-IL pattern exposure followed by a UV-flood exposure (at different doses depending on the PSCAR tested) using a light emitting diode (LED) lamp $(\lambda=365 \mathrm{~nm})$ adapted on-site at PSI.

We have first tested a PSCAR resist (PSCAR1) derived from the high performing reference resist R1 at different UV-flood exposure doses. PSCAR1 is resolved down to $16 \mathrm{~nm}$ at all UV-flood exposures tested without EL (not shown here). Fig. 8 shows the SEM (L/S) images of the produced patterns at $18 \mathrm{~nm}$ resolution as the UV-flood exposure 
increases from $0 \mathrm{~J} / \mathrm{cm}^{2}, 0.25 \mathrm{~J} / \mathrm{cm}^{2}$ and $0.5 \mathrm{~J} . \mathrm{cm}^{2}$. Table 3 shows the corresponding BE, LWR, EL and $Z$-values obtained from those exposures. The sensitivity improves (up to $40 \%$ ) with increasing UV-flood exposure (from 0 to $0.5 \mathrm{~J} / \mathrm{cm}^{2}$ ) dose at $18 \mathrm{~nm}$ HP. Nonetheless, the EL decreases and the LWR increases as well with increasing UV-flood exposure.

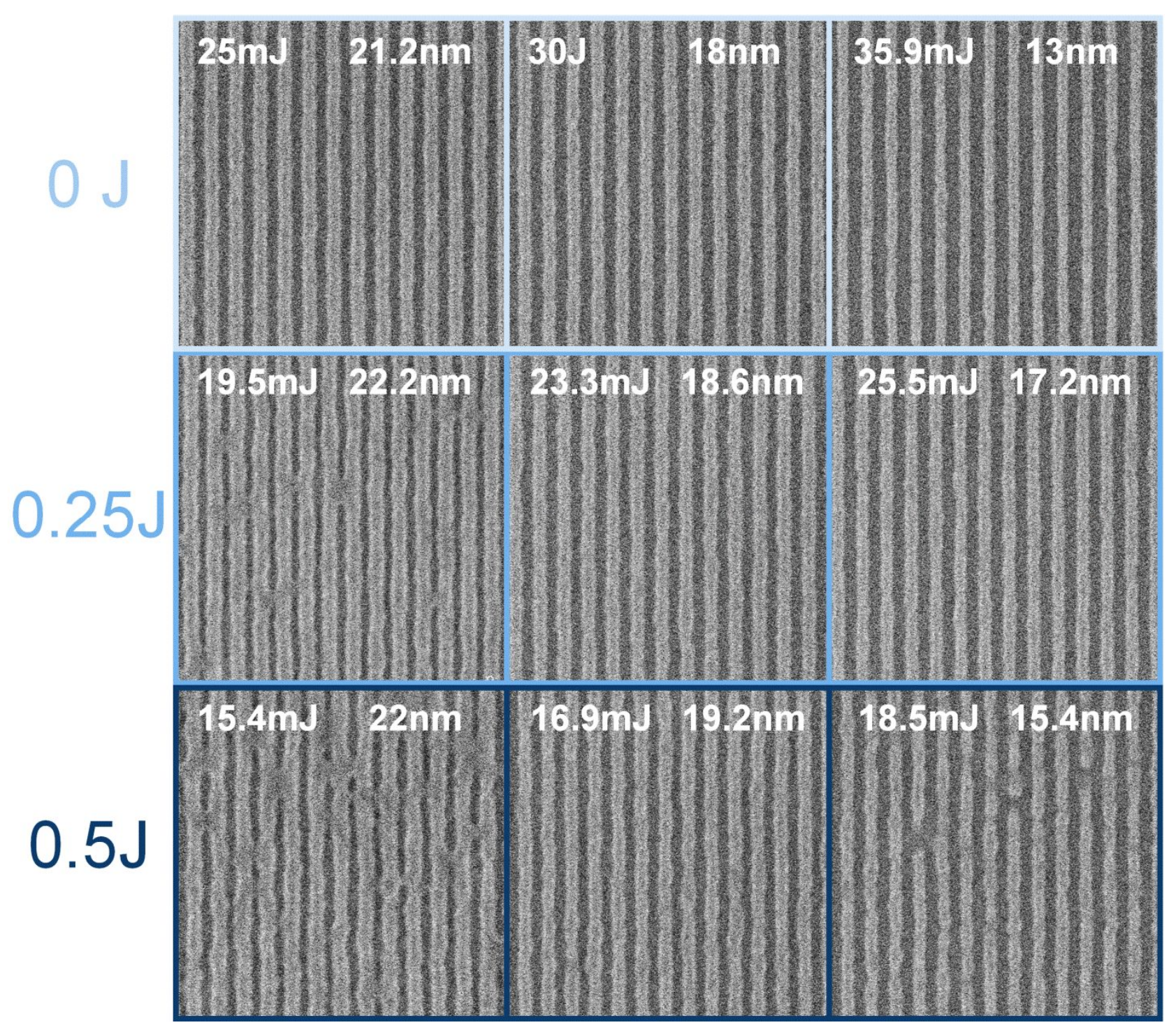

Figure 8. SEM (L/S) images of the produced patterns with PSCAR1 at $18 \mathrm{~nm}$ resolution as the UV-flood exposure increases from $0 \mathrm{~J} / \mathrm{cm}^{2}, 0.25 \mathrm{~J} / \mathrm{cm}^{2}$ and $0.5 \mathrm{~J} / \mathrm{cm}^{2}$.

Table 3. BE, EL, LWR and Z-factors obtained as the UV-flood exposure dose increases while using PSCAR1 at $\mathrm{HP}=18 \mathrm{~nm}$.

\begin{tabular}{|c|c|c|c|c|}
\hline $\begin{array}{l}\text { UV-flood } \\
\text { dose (J) }\end{array}$ & $\begin{array}{c}\text { EUV BE } \\
(\mathrm{mJ} / \mathrm{cm} 2)\end{array}$ & EL (\%) & $\begin{array}{l}\text { LWR } \\
(\mathrm{nm})\end{array}$ & z-factor \\
\hline 0 & 29.5 & 17 & 3.9 & $1.3 \mathrm{E}-08$ \\
\hline 0.25 & 24.4 & 14 & 4.6 & $1.5 \mathrm{E}-08$ \\
\hline 0.5 & 17.3 & 1 & 6.1 & $1.8 \mathrm{E}-08$ \\
\hline
\end{tabular}


In order to address this issue, a second PSCAR resist (PSCAR2) derived from the high resolution and high sensitivity resist R2 was tested. The formulation of this resist also contains a quencher for contrast improvement. PSCAR2 is resolved down to $14 \mathrm{~nm}$ at all UV-flood exposures tested without EL. Fig. 9 shows the SEM (L/S) images of the produced patterns at $16 \mathrm{~nm}$ resolution as the UV-flood exposure increases from $0 \mathrm{~J} / \mathrm{cm}^{2}, 1 \mathrm{~J} / \mathrm{cm}^{2}$ and $2 \mathrm{~J} / \mathrm{cm}^{2}$. Table 4 shows the corresponding BE, LWR, EL and $Z$-values obtained from those exposures. We also observe a sensitivity improvement (up to $\sim 7 \%$ ) with increasing UV-flood exposure dose at $16 \mathrm{~nm}$ HP. Though, the LWR and EL remain almost unchanged with increasing UV-flood exposure dose, the sensitivity improvement is only minor and must be enhanced for future commercial successes of this process. Further resist and process optimization therefore still ongoing.
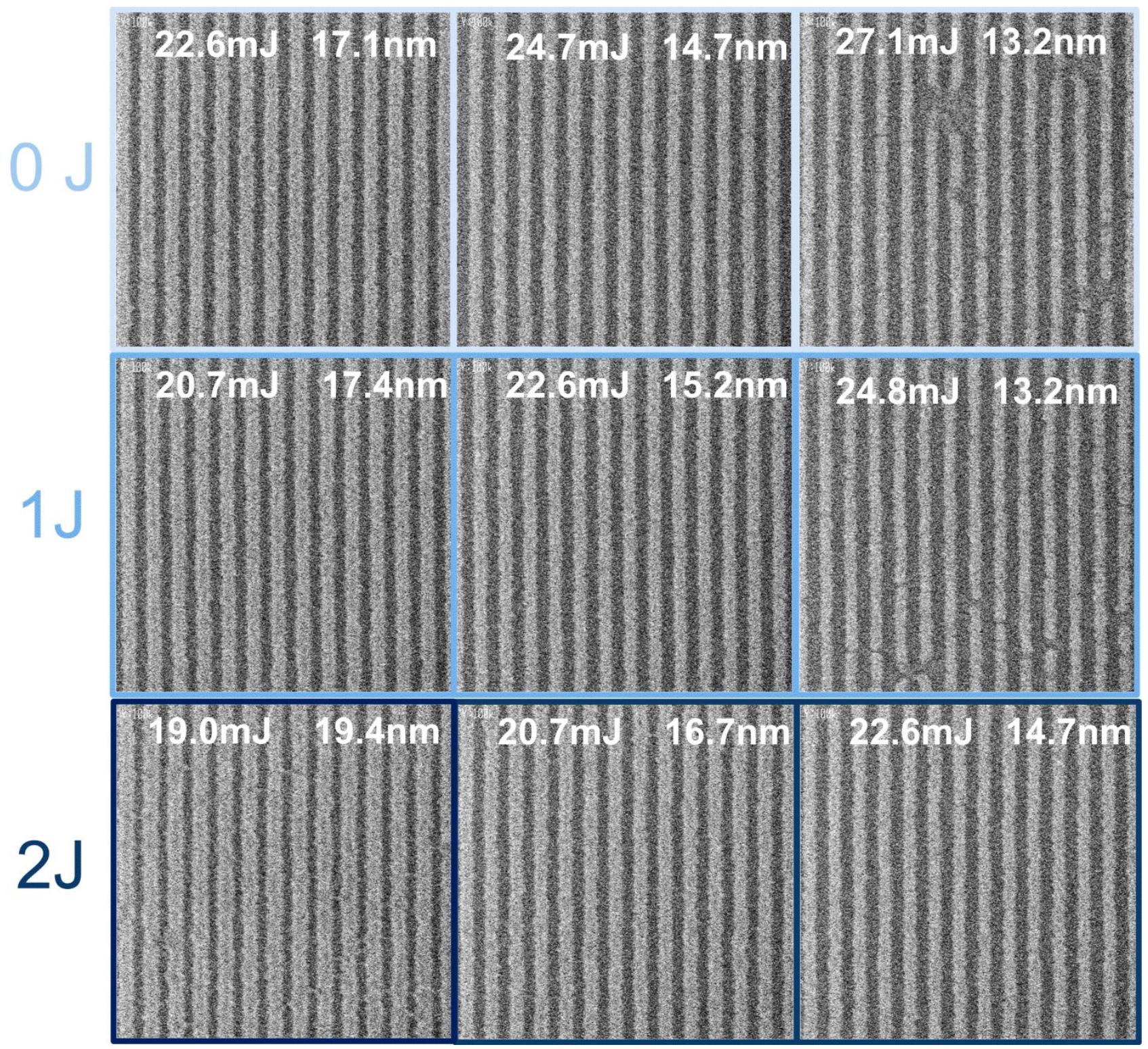

Figure 9. SEM (L/S) images of the produced patterns with PSCAR2 at $18 \mathrm{~nm} \mathrm{HP}$ as the UV-flood exposure increases from $0 \mathrm{~J} / \mathrm{cm}^{2}, 1 \mathrm{~J} / \mathrm{cm}^{2}$ and $2 \mathrm{~J} / \mathrm{cm}^{2}$. 
Table 4. BE, EL, LWR and Z-factors obtained as the UV-flood exposure dose increases while using PSCAR2 at $\mathrm{HP}=16 \mathrm{~nm}$.

\begin{tabular}{|c|c|c|c|c|}
\hline $\begin{array}{c}\text { UV-flood } \\
\text { dose }(\mathrm{J})\end{array}$ & $\begin{array}{c}\text { *EUV BE } \\
(\mathrm{mJ} / \mathrm{cm} 2)\end{array}$ & EL (\%) & $\begin{array}{c}\text { LWR } \\
(\mathrm{nm})\end{array}$ & z-factor \\
\hline 0 & 23.4 & 9 & 4.8 & $2.2 \mathrm{E}-08$ \\
\hline 1 & 22.3 & 9 & 4.8 & $2.1 \mathrm{E}-08$ \\
\hline 2 & 21.7 & 9 & 4.9 & $2.0 \mathrm{E}-08$ \\
\hline
\end{tabular}

\section{CONCLUSIONS}

In this study we have identified several promising candidates that simultaneously meet sensitivity, LWR and EL high performance requirements (arbitrary high performance threshold values, not set by industry) with the aim of resolving line space (L/S) features for the 7 and $5 \mathrm{~nm}$ logic node $(16 \mathrm{~nm}$ and $13 \mathrm{~nm} \mathrm{HP}$, respectively) for future investigation. In addition, several very promising CARs (UL1R1, UL1R9 and UL1R10) were found to be well resolved down to $12 \mathrm{~nm}$ and $11 \mathrm{~nm}$ HP with minimal pattern collapse and bridging, a remarkable feat for CARs. Finally, the performance of two negative tone state-of-the-art alternative resist platforms previously investigated was compared to the CAR performance at and below $16 \mathrm{~nm} \mathrm{HP}$ resolution, demonstrating the need for alternative resist solutions at $13 \mathrm{~nm}$ resolution and below.

In this work, we furthermore have presented promising early proof-of-principle results for PSCAR $\mathrm{TM}_{\mathrm{TM}}$ a multi-exposure lithography process. A minor sensitivity improvement of $7 \%$ with a UV-flood exposure dose of $2 \mathrm{~J} / \mathrm{cm}^{2}$ was shown for the PSCAR2 system while the LWR and EL remain almost unchanged with increasing UV-flood exposure dose. Nonetheless, further development still needed and is ongoing in order to fully demonstrate the potential of PSCAR for sensitivity improvement while other important performance characteristics are not being compromised.

\section{ACKNOWLEDGEMENTS}

We would like to acknowledge Markus Kropf, Roberto Fallica, and Daniel Fan for their technical expertise and assistance. We thank our various materials suppliers greatly. Part of this work was performed at the Swiss Light Source in the Paul Scherrer Institute.

\section{REFERENCES}

[1] Brink, M. v. d. "Cost-effective shrink with Holistic Lithography, extended by EUV," Proc. EUVL Symposium, Maastricht, The Neatherlands, Maastricht, The Neatherlands, (2015).

[2] Khare, M., "A New Wave of Innovation in Computer Chips," IBM Research, (2015).

[3] Buitrago, E.; Yildirim, O.; Fallica, R., et al. "The road towards single digit nanometer resolution patterning in mass production: State-of-the-art EUV resists platforms compared," Proc. EUVL Symposium, Maastricht, The Neatherlands, Maastricht, The Neatherlands, (2015).

[4] Frommhold, A.; Brown, A. G.; Palmer, R. E., et al. "Organic hard masks utilizing fullerene derivatives," Proc. SPIE Advanced Lithography, San Jose, San Jose, pp 94250L-94250L-8, (2015).

[5] Tagawa, S.; Enomoto, S.; Oshima, A., "Super high sensitivity enhancement by photo-sensitized chemically amplified resist (PS-CAR) process," Journal of Photopolymer Science and Technology,26 (6), 825-830, (2013).

[6] Tagawa, S.; Oshima, A.; Enomoto, S., et al. "High-resist sensitization by pattern and flood combination lithography," Proc. SPIE Advanced Lithography, International Society for Optics and Photonics: pp 90481S-90481S-6, (2014).

[7] Ekinci, Y.; Vockenhuber, M.; Hojeij, M., et al. "Evaluation of EUV resist Performance with Interference Lithography Towards $11 \mathrm{~nm}$ Half-Pitch and Beyond," Proc. SPIE Advanced Lithography, San Jose, San Jose, pp 867910-867910-11, (2013).

[8] Mojarad, N.; Fan, D.; Gobrecht, J., et al., "Broadband Interference Lithography at Extreme Ultraviolet and Soft X-ray Wavelengths," Optics Letters, 39 (8), 2286-2289, (2014).

[9] Buitrago, E.; Yildirim, O.; Verspaget, C., et al. "Evaluation of EUV Resist Performance Using Interference Lithography," Proc. SPIE Advanced Lithography, pp 94221S-94221S-13, (2015). 
[10] Solak, H. H.; Ekinci, Y.; Kaser, P., et al., "Photon-beam lithography reaches 12.5nm half-pitch resolution," Journal of Vacuum Science \& Technology B: Microelectronics and Nanometer Structures, 25 (1), 91-95, (2007).

[11] Päivänranta, B.; Langner, A.; Kirk, E., et al., "Sub-10 nm Patterning Using EUV Unterference Lithography," Nanotechnology,22 (37), 375302, (2011).

[12] Buitrago, E.; Fallica, R.; Fan, D., et al., "SnOx high-efficiency EUV interference lithography gratings towards the ultimate resolution in photolithography," Microelectronic Engineering, 155, 44-49, (2016).

[13] Ekinci, Y.; Vockenhuber, M.; Terhalle, B., et al. "Evaluation of resist performance with EUV interference lithography for sub-22-nm patterning," Proc. SPIE Advanced Lithography, pp 83220W-83220W-11, (2012).

[14] Wallow, T.; Higgins, C.; Brainard, R., et al. "Evaluation of EUV resist materials for use at the $32 \mathrm{~nm}$ half-pitch node," Proc. SPIE Advanced Lithography, pp 69211F-69211F-11, (2008).

[15] Ito, H.; Grant Willson, C.; Frechet, J. H. J., "New UV Resists with Negative or Positive Tone," In Symposium on VLSI Technology, pp 86-87, (1982).

[16] Putna, E. S.; Younkin, T. R.; Caudillo, R., et al. "EUV Lithography for 22nm Half Pitch and Beyond: Exploring Resolution, LWR, and Sensitivity Tradeoffs," Proc. SPIE Advanced Lithography, pp 76360P-76360P-7, (2010). 